\title{
On isotropic cylindrically symmetric stellar models
}

\author{
Brien C Nolan and Louise V Nolan \\ School of Mathematical Sciences, Dublin City University, Glasnevin, Dublin 9, \\ Ireland \\ E-mail: brien.nolan@dcu.ie, louise.nolan3@mail.dcu.ie
}

\begin{abstract}
We attempt to match the most general cylindrically symmetric vacuum space-time with a Robertson-Walker interior. The matching conditions show that the interior must be dust filled and that the boundary must be comoving. Further, we show that the vacuum region must be polarized. Imposing the condition that there are no trapped cylinders on an initial time slice, we can apply a result of Thorne's and show that trapped cylinders never evolve. This results in a simplified line element which we prove to be incompatible with the dust interior. This result demonstrates the impossibility of the existence of an isotropic cylindrically symmetric star (or even a star which has a cylindrically symmetric portion). We investigate the problem from a different perspective by looking at the expansion scalars of invariant null geodesic congruences and, applying to the cylindrical case, the result that the product of the signs of the expansion scalars must be continuous across the boundary. The result may also be understood in relation to recent results about the impossibility of the static axially symmetric analogue of the Einstein-Straus model.
\end{abstract}

PACS numbers: 04.20.Dw, 04.20.Jb, 04.40.Dg 


\section{Introduction}

In this article, we study non-spherical gravitational collapse. This is an area where not much is currently known in the highly non-spherical regime, where perturbative studies are not appropriate: this is in contrast to the near-spherical case. To study this physical situation, mathematical assumptions must be made. Ideally, one would like to study dynamical axially symmetric situations, leading to PDE's in $2+1$ dimensions. To attempt to capture the flavour of possible outcomes and to pose tractable problems, a further symmetry is added (translational symmetry along an axis), leading to cylindrical symmetry. This models highly prolate collapse and as mentioned below, can be used to construct axially symmetric models by the addition of hemispherical caps to a finite cylindrical object.

Cylindrically symmetric space-times in General Relativity are idealized models, but they provide insight into non-spherical gravitational collapse and the non-linearity of the field equations. Key results in this field include the following: Thorne showed that horizons cannot evolve in the vacuum region surrounding a collapsing infinite cylinder [1]. Berger, Chrusciel and Moncrief proved that asymptotic flatness, energy conditions and cylindrical symmetry exclude the existence of compact trapped surfaces 2]. Apostolatos and Thorne proved that even an infinitesimal amount of rotation can halt the collapse of an infinite cylindrical dust shell [3]. More recent work describes the cylindrically symmetric collapse of an infinite null dust shell [4, non-rotating, infinite dust cylinders [5], 6], counter-rotating dust shells [7, [8, 9] and self-similar scalar field [10]. These, and other results furnish us with a clearer picture of non-spherical collapse in which gravitational radiation, angular momentum and critical phenomena play an important role.

In many instances these results are in sharp contrast to results for the corresponding spherically symmetric model, for example Berger et al's strong cosmic censorship result 2] and Thorne's [1] and Golcalves' [1] results ruling out certain types of horizon. So with a view to comparing and contrasting cylindrical and spherical collapse, we investigate a cylindrical version of the "standard" model of spherical collapse, the Oppenheimer-Snyder model [12]. However unlike that model we do not use the a priori assumption that the interior comprises pressureless dust. In this paper we apply the standard matching techniques, without any conditions of staticity, to the cylindrically symmetric case to obtain a general result about the evolution of cylindrically symmetric objects in a vacuum space-time.

As a preliminary we examine a general matching of a space-time $\left(\mathcal{V}^{-}, g^{-}\right)$to a vacuum space-time $\left(\mathcal{V}^{+}, g^{+}\right)$, with the matching condition [13],

$$
\left[T_{\mu \nu}\right] n^{\nu}=\left(T_{\mu \nu}^{+}-T_{\mu \nu}^{-}\right) n^{\nu}=0,
$$

where $T_{\mu \nu}^{ \pm}$is the energy momentum tensor in $\mathcal{V}^{ \pm}$respectively. The conditions (11) are sometimes known as the Israel junction conditions and follow from the standard (Darmois) matching conditions of General Relativity - continuity of the first and second 
fundamental forms - which are used throughout. For vacuum $\mathcal{V}^{+}$, (11) implies that

$$
T_{\mu \nu}^{-} n^{\nu}=0
$$

on the matching hypersurface $\Sigma$. Assuming that the energy momentum tensor of $\left(\mathcal{V}^{-}, g^{-}\right)$is that of a perfect fluid, we have

$$
T_{\mu \nu}^{-}=(p+\rho) u_{\mu} u_{\nu}+p g_{\mu \nu},
$$

where $u_{\mu}$ is a unit future pointing time-like vector. Then (2) becomes

$$
p n_{\mu}+(p+\rho) n_{\nu} u^{\nu} u_{\mu}=0 .
$$

If we invoke the weak energy condition, $\rho \geq 0$ and $\rho+p \geq 0$, and require $\rho \neq 0$ to avoid a trivial case, then (3) implies that $p=0$ on $\Sigma$ and $u_{\nu} n^{\nu}=0$. In other words, matching with vacuum can only be done across a time-like hypersurface on which the pressure vanishes. We are considering the case where $\left(\mathcal{V}^{-}, g^{-}\right)$is a Robertson-Walker (RW) space-time. Since the pressure of such a space-time is homogeneous, this implies that the pressure must vanish everywhere. So we will consider a RW interior space-time $\left(\mathcal{V}^{-}, g^{-}\right)$with cylindrically symmetric line element in coordinates $\{t, \rho, x, \varphi\}$

$$
d s_{-}^{2}=-d t^{2}+a^{2}(t)\left(d \rho^{2}+\Upsilon_{, \rho}^{2}(\rho, \epsilon) d x^{2}+\Upsilon^{2}(\rho, \epsilon) d \varphi^{2}\right)
$$

where $a(t)$ is the scale factor and for collapsing dust

$$
a(t)=a_{0}|t|^{2 / 3}, \quad t \in(-\infty, 0],
$$

and where $\Upsilon(\rho, \epsilon)$ satisfies

$$
\Upsilon(\rho, \epsilon)= \begin{cases}\sinh \rho, & \epsilon=-1, \\ \rho, & \epsilon=0, \\ \sin \rho, & \epsilon=+1,\end{cases}
$$

and where $\epsilon$ is the curvature index so that $\epsilon=1,0,-1$ for closed, flat or open RW models, respectively. We will match to a general cylindrically symmetric unpolarized vacuum exterior space-time $\left(\mathcal{V}^{+}, g^{+}\right)$given by the line element in coordinates $\{T, R, Z, \Phi\}$ [14] (see also [15, 16])

$$
d s_{+}^{2}=e^{2(\gamma-\psi)}\left(-d T^{2}+d R^{2}\right)+e^{2 \psi}(d Z+\omega d \Phi)^{2}+\alpha^{2} e^{-2 \psi} d \Phi^{2},
$$

and $\gamma, \psi, \omega$ and $\alpha$ are functions of $T$ and $R \ddagger$. In $\oint 2$ we describe the most general matching hypersurface. In $\$ 3$ by assuming that $\omega(R, T)$ is analytic in $R$ and $T$ on $\Sigma$ we show that $\omega=0$ everywhere, i.e. that an unpolarized vacuum space-time cannot be matched to a dust interior. In $₫$ we show that, by applying an argument due to Thorne and requiring that there are no trapped surfaces initially, we can reduce to the case $\alpha(R, T)=R$. In $\$ 5$, we show that the matching of the (now simplified) exterior to the $\ddagger$ The intention is that if the matching were successful we would propose a global picture of the whole matched space-time with the infinite RW cylinder truncated at two values of $z$ and hemispherical caps inserted at these points $z=z_{1}$ and $z=z_{2}$ so that the RW portion corresponds to $z_{1}<z<z_{2}$ [17. Thus the vacuum region should include the axis beyond the caps. This implies orthogonal transitivity of the isometry group and so (6) applies. [15] 
RW interior is impossible. We also show that continuity of the metric alone rules out the existence of $\Sigma$. Thus one cannot match the RW interior with the polarized vacuum exterior even if there is a distributional shell of matter forming the boundary. This demonstrates the impossibility of matching a RW interior to a cylindrically symmetric vacuum exterior.

\section{Matching hypersurface}

We begin with the following coordinate systems

$$
\begin{array}{ll}
\text { Interior } \mathcal{V}^{-}: x_{-}^{\mu} & =\{t, \rho, x, \varphi\}, \\
\text { Exterior } \mathcal{V}^{+}: x_{+}^{\mu} & =\{T, R, Z, \Phi\}, \\
\text { Matching hypersurface } \Sigma: \xi^{a} & =\{\tau, z, \phi\} .
\end{array}
$$

We use the following conventions: $\mu, \nu, . .=0,1,2,3$ and $a, b, . .=1,2,3$, with prime and overdot referring to differentiation with respect to $T$ and $t$ respectively. In order to have a description of $\Sigma^{ \pm}$, the hypersurface in $\mathcal{V}^{ \pm}$respectively $\left(\Sigma^{ \pm} \subset \mathcal{V}^{ \pm}\right)$, in terms of the coordinates $\{\tau, z, \phi\}$ we proceed as follows [18]. A careful choice of coordinate $\phi$, use of the commutativity of the Killing vectors in $\mathcal{V}^{+}$, observation of the uniqueness of the generator of axial symmetry and a coordinate transformation $(Z, \Phi) \rightarrow(\tilde{Z}(Z), \tilde{\Phi}(Z, \Phi))$ that does not alter the form of the line element in $\mathcal{V}^{+}$are all needed to arrive at the following description of $\Sigma^{ \pm}$

$$
\begin{aligned}
& \Sigma^{-}:\left\{t=\tau, \rho=\rho_{0}, x=z, \varphi=\phi\right\}, \\
& \Sigma^{+}:\left\{T=T_{0}(t), R=R_{0}(t), Z=z, \Phi=\phi\right\},
\end{aligned}
$$

with the requirement $0<\rho_{0}$. In theory four different matchings of $\left(\mathcal{V}^{-}, g^{-}\right)$and $\left(\mathcal{V}^{+}, g^{+}\right)$ are possible, depending on the choice of continuous normal $\vec{n}^{ \pm}$to $\Sigma^{ \pm}$in $\mathcal{V}^{ \pm}$. However our aim is to describe a space-time consisting of a RW interior and a vacuum exterior. For $\vec{n}^{-}$, we choose the normal to point toward cylinders of increasing radius. For $\vec{n}^{+}$, we want to do the same. This requires that $\vec{n}^{+}$points towards larger values of $\alpha$. The coordinate $R$ has yet to be specified and it may happen that either $\alpha_{, R}>0$ or $\alpha_{, R}<0$. We assume further that the axis of the vacuum space-time resides in the region removed to accommodate the RW portion. Thus $\alpha_{, R}<0$ in $\mathcal{V}^{+}$can only come about if $R$ decreases away from $\Sigma^{+}$. Hence the $\alpha_{, R}<0$ case can be converted to the $\alpha_{, R}>0$ case by a coordinate transformation of the form

$$
R \rightarrow \hat{R}=R^{*}-R \text {. }
$$

Thus in the coordinates of (8), we will assume that $\vec{n}^{+}$points in the direction of increasing $R$. We will refer to this arrangement of $\Sigma^{ \pm}$and $\vec{n}^{ \pm}$by saying that $\vec{n}$ points out of $\mathcal{V}^{-}$and into $\mathcal{V}^{+}$. 
On isotropic cylindrically symmetric stellar models

\section{Reduction to the polarized case}

We begin with the line element (6). The tangent vectors, $e_{a}^{\mu}=\frac{\partial x^{\mu}}{\partial \xi^{a}}$, to the hypersurface, $\Sigma$ are

$$
\begin{aligned}
e_{1}^{\mu} & =\left(\frac{\partial T}{\partial t}, \frac{\partial R}{\partial t}, 0,0\right), \\
e_{2}^{\mu} & =(0,0,1,0), \\
e_{3}^{\mu} & =(0,0,0,1),
\end{aligned}
$$

so that

$$
\vec{e}_{1}=e_{1}^{\mu} \frac{\partial}{\partial x^{\mu}}=\frac{d T}{d t}\left(\frac{\partial}{\partial T}+\left(R_{0}^{\prime}\right) \frac{\partial}{\partial R}\right) .
$$

Thus there is a tangential derivative proportional to

$$
\frac{\partial}{\partial T}+\left(R_{0}^{\prime}\right) \frac{\partial}{\partial R}
$$

which for convenience we will refer to as the tangential derivative. (The other tangential derivatives, $\partial_{\phi}, \partial_{z}$, are trivial in the sense that they play no role in the dynamics.) We will require the following matching equations:

$$
\begin{aligned}
& h_{z \phi}^{+} \stackrel{\Sigma}{=} h_{z \phi}^{-} \Leftrightarrow \omega_{\Sigma} \stackrel{\Sigma}{=} 0, \\
& h_{\tau \tau}^{+} \stackrel{\Sigma}{=} h_{\tau \tau}^{-} \Rightarrow\left(1-\left(R_{0}^{\prime}\right)^{2}\right)>0, \\
& K_{z \phi}^{+} \stackrel{\Sigma}{=} K_{z \phi}^{-} \Leftrightarrow\left(\left(R_{0}^{\prime}\right) \frac{\partial \omega}{\partial T}+\frac{\partial \omega}{\partial R}\right) \stackrel{\Sigma}{=} 0,
\end{aligned}
$$

where $h_{a b}$ is the first fundamental form, $K_{a b}$ is the second fundamental form and we use $\stackrel{\Sigma}{=}$ to indicate equality on $\Sigma$. We can take the tangential derivative of $\omega$ and then evaluate it on $\Sigma$

$$
\left(\frac{\partial \omega}{\partial T}+\left(R_{0}^{\prime}\right) \frac{\partial \omega}{\partial R}\right) \stackrel{\Sigma}{=} 0 .
$$

By our matching condition (11) this implies

$$
\frac{\partial \omega}{\partial T}\left(1-\left(R_{0}^{\prime}\right)^{2}\right) \stackrel{\Sigma}{=} 0 .
$$

Using the matching conditions (10) then gives the result

$$
\Rightarrow \frac{\partial \omega}{\partial T} \stackrel{\Sigma}{=} 0, \quad \frac{\partial \omega}{\partial R} \stackrel{\Sigma}{=} 0,
$$

or equivalently

$$
\omega^{(1)} \stackrel{\Sigma}{=} 0,
$$

where $\omega^{(k)}$ denotes all partial derivatives of $\omega$ of order $k$.

It is then straightforward to show that $\omega^{(2)} \stackrel{\Sigma}{=} 0$ by considering the field equation (A.1), which we can write in the form

$$
\frac{\partial^{2} \omega}{\partial T^{2}}-\frac{\partial^{2} \omega}{\partial R^{2}}=f\left(\omega, \omega^{(1)}\right)
$$


where $f$ is some polynomial function satisfying $f(0,0)=0$, which by (9) and (13) equals zero when evaluated at $\Sigma$. By taking tangential derivatives of both of (13) and comparing with (14), we obtain the result $\omega^{(2)}=0$.

In like manner, we can show that if $\omega$ is $C^{k}, k \leq \infty$ on a neighbourhood of $\Sigma$, then $\omega^{(k)} \stackrel{\Sigma}{=} 0$. We use an induction argument. If we assume that $\omega^{(j)} \stackrel{\Sigma}{=} 0$ for $0 \leq j \leq k$ is true then by proving $\omega^{(k+1)} \stackrel{\Sigma}{=} 0$ is true and using (13) we have the desired result.

To show that $\omega^{(k+1)} \stackrel{\Sigma}{\equiv} 0$ is true, we take the tangential derivative of our assumed $\omega^{(k)} \stackrel{\Sigma}{=} 0$. There are $(k+1)$ of these tangential derivative equations, and they are of the form

$$
\begin{array}{ll}
\frac{\partial^{k+1} \omega}{\partial T^{k+1}}+R_{0}^{\prime} \frac{\partial^{k+1} \omega}{\partial R \partial T^{k}} & \stackrel{\Sigma}{=} 0, \\
\frac{\partial^{k+1} \omega}{\partial T^{k} \partial R}+R_{0}^{\prime} \frac{\partial^{k+1} \omega}{\partial R^{2} \partial T^{k-1}} \quad \stackrel{\Sigma}{=} 0, \\
\frac{\partial^{k+1} \omega}{\partial T^{k-1} \partial R^{2}}+R_{0}^{\prime} \frac{\partial^{k+1} \omega}{\partial R^{3} \partial T^{k-2}} \stackrel{\Sigma}{=} 0 \quad \text { etc. }
\end{array}
$$

Then we consider the field equation (14). Taking successive partial derivatives of (14) we have

$$
\frac{\partial^{k+1} \omega}{\partial T^{k+1}}-\frac{\partial^{k+1} \omega}{\partial T^{k-1} \partial R^{2}}=F\left(\omega, \cdots, \omega^{(k)}\right),
$$

and the form of $f$ in (A.1) shows that $F(0, \cdots, 0)=0$. But evaluated on $\Sigma$ we know by assumption that

$$
\omega^{(j)} \stackrel{\Sigma}{=} 0 \text { for } 0 \leq j \leq k
$$

and therefore

$$
\frac{\partial^{k+1} \omega}{\partial T^{k+1}}-\frac{\partial^{k+1} \omega}{\partial T^{k-1} \partial R^{2}} \stackrel{\Sigma}{=} 0 .
$$

This equation together with (15) and (16) gives the relation

$$
\frac{\partial^{k+1} \omega}{\partial T^{k} \partial R}\left(1-\left(R_{0}^{\prime}\right)^{2}\right) \stackrel{\Sigma}{=} 0,
$$

and so by (10)

$$
\frac{\partial^{k+1} \omega}{\partial T^{k} \partial R} \stackrel{\Sigma}{=} 0
$$

Substituting this equation into the appropriate tangential equation shows, by a cascade effect, each partial derivative of order $(k+1)$ to be zero when evaluated at $\Sigma$, proving our assertion. We can then write down the following lemma.

Lemma 1 If $\omega$ is analytic on a neighbourhood $\Omega$ of $\Sigma$, then $\omega \equiv 0$ on $\Omega$.

Proof: Let $\left(R_{1}, T_{1}\right) \in \Omega$. Then we can write $R_{1}=R_{0}(T)+R_{*}, T_{1}=T+T_{*}$ for some numbers $R_{*}, T_{*}$ and where $\left(R_{0}(T), T\right) \in \Sigma$. By analyticity, we can write

$$
\omega\left(R_{0}(T)+R_{*}, T+T_{*}\right)=\left[\sum_{n=1}^{\infty} \frac{1}{n !}\left(T_{*} \frac{\partial}{\partial T}+R_{*} \frac{\partial}{\partial R}\right)^{n} \omega\right]_{\Sigma} .
$$

The result follows immediately. 
On isotropic cylindrically symmetric stellar models

Therefore, assuming that $\omega(R, T)$ is an analytic function we see that $\omega=0$ on a neighbourhood of $\Sigma$ by using the matching conditions and the vacuum field equations. So we have henceforth that $\omega=0$ and (6) becomes

$$
d s_{+}^{2}=e^{2(\gamma-\psi)}\left(-d T^{2}+d R^{2}\right)+e^{2 \psi} d Z^{2}+\alpha^{2} e^{-2 \psi} d \Phi^{2} .
$$

\section{Further simplification of the vacuum line element}

We note that the general solution of the vacuum field equation

$$
\frac{\partial^{2} \alpha}{\partial R^{2}}-\frac{\partial^{2} \alpha}{\partial T^{2}}=0
$$

is

$$
\alpha(T, R)=F(U)+G(V),
$$

where $U=T-R$ and $V=T+R$. Following Thorne [3] we define the character of space-time with line element (19) at any event $p$ to be $D^{(+)}$if $\nabla \alpha$ is space-like and points away from the symmetry axis, $D^{(-)}$if $\nabla \alpha$ is space-like and points toward the symmetry axis, $D^{(0 \uparrow)}$ if $\nabla \alpha$ is time-like and points toward the future, and $D^{(0 \downarrow)}$ if $\nabla \alpha$ is time-like and points toward the past. So at any event $p$, the character is

$$
\begin{aligned}
D^{(+)} & \Leftrightarrow \frac{\partial F}{\partial U}<0, \frac{\partial G}{\partial V}>0, \\
D^{(-)} & \Leftrightarrow \frac{\partial F}{\partial U}>0, \frac{\partial G}{\partial V}<0, \\
D^{(0 \uparrow)} & \Leftrightarrow \frac{\partial F}{\partial U}>0, \frac{\partial G}{\partial V}>0, \\
D^{(0 \downarrow)} & \Leftrightarrow \frac{\partial F}{\partial U}<0, \frac{\partial G}{\partial V}<0 .
\end{aligned}
$$

It is straightforward to show that $D^{(+)}$and $D^{(-)}$at $p$ imply no trapped cylinders at $p$, while $D^{(0 \uparrow)}$ and $D^{(0 \downarrow)}$ implies trapping. To prove this we note that the standard line element (19) can be rewritten in terms of null coordinates $U$ and $V$

$$
d s_{+}^{2}=-e^{2(\gamma-\psi)} d U d V+e^{2 \psi} d Z^{2}+\alpha^{2} e^{-2 \psi} d \Phi^{2} .
$$

The condition for a two-cylinder, $S$, of constant $T$ and $R$ to be untrapped is that

$$
\theta_{+} \theta_{-}<0
$$

where $\theta_{ \pm}$are respectively the expansions of the future-pointing outgoing and ingoing null geodesics $l_{ \pm}^{\mu}$ orthogonal to $S$, given by

$$
\begin{aligned}
& l_{+}^{\mu}=a(V) e^{-2(\gamma-\psi)} \delta_{U}^{\mu}, \\
& l_{-}^{\mu}=b(U) e^{-2(\gamma-\psi)} \delta_{V}^{\mu},
\end{aligned}
$$

where $a(V)>0$ and $b(U)>0$. We find the expansions of these null geodesics to be

$$
\begin{aligned}
& \theta_{+}:=\nabla_{\mu} l_{+}^{\mu}=a(V) e^{-2(\gamma-\psi)} \frac{\alpha_{, U}}{\alpha}, \\
& \theta_{-}:=\nabla_{\mu} l_{-}^{\mu}=b(U) e^{-2(\gamma-\psi)} \frac{\alpha_{, V}}{\alpha},
\end{aligned}
$$


and we can write

$$
\theta_{+} \theta_{-}=a(V) b(U) e^{-4(\gamma-\psi)} \frac{\alpha_{, V} \alpha_{, U}}{\alpha^{2}}=a(V) b(U) \frac{e^{-4(\gamma-\psi)}}{\alpha^{2}} \frac{d F}{d U} \frac{d G}{d V} .
$$

The result follows by inspection.

We require that initially there are no trapped surfaces

$$
D^{(+)} \text {or } D^{(-)} \text {at } T=0 .
$$

However we note that in the absence of trapped cylinders

$$
\begin{aligned}
D^{(+)} & \Leftrightarrow \frac{\partial \alpha}{\partial R}>0, \\
D^{(-)} & \Leftrightarrow \frac{\partial \alpha}{\partial R}<0 .
\end{aligned}
$$

These constraints, together with the assumptions of $\S 2$ rule out $D^{(-)}$

$$
\Rightarrow D^{(+)} \text {at } T=0 \text {. }
$$

Thorne [3] showed that in the vacuum region outside a cylindrical shell of matter, with the constraint (24), the only possible character change is

$$
D^{(+)} \rightarrow D^{(0 \downarrow)} \text {. }
$$

However this happens on an ingoing null hypersurface which would intersect $T=0$

$$
\Rightarrow D^{(0 \downarrow)} \text { at } T=0 \text { for } R>R_{*},
$$

where $R_{*} \geq 0$. The contradiction between equations (24) and (25) implies that

$$
D^{(+)} \text {at } T=0 \Rightarrow D^{(+)} \forall T \geq 0 \text {. }
$$

The argument also holds in the vacuum region outside our cylindrical star.

Furthermore it has been shown [19] that in a space-time of character $D^{(+)}$we can make a coordinate transformation

$$
(T, R) \rightarrow(\hat{T}(T, R), \hat{R}(T, R))
$$

whereby $\alpha(R, T)$ becomes the new radial variable $\hat{R}$. Therefore if our vacuum space-time does not contain trapped cylinders initially and is not radially closed $[3]\left(D^{(+)}\right.$at $\left.T=0\right)$ we can use the above results to describe the vacuum exterior space-time, $\left(\mathcal{V}^{+}, g^{+}\right)$, by

$$
d s_{+}^{2}=-e^{2(\gamma-\psi)}\left(d T^{2}-d R^{2}\right)+e^{2 \psi} d Z^{2}+R^{2} e^{-2 \psi} d \Phi^{2},
$$

where we have rewritten $\hat{T}$ and $\hat{R}$ as $T$ and $R$ without confusion.

\section{Impossibility of the matching}

Thus far, we have shown that the most general matching of a non-vacuum RW universe with a vacuum cylindrically symmetric space-time reduces to the case where the RW universe is dust-filled, the boundary is co-moving, the vacuum region is polarized and has character $D^{(+)}$. In this section, we show that this matching configuration is impossible. More generally, we show that metric matching alone rules out the matching 
of a collapsing RW universe across a co-moving hypersurface with a polarized cylindrical vacuum space-time. Thus even the insertion of a distributional shell of matter (which would arise in the case where $K_{\mu \nu}$ is discontinuous) cannot yield a solution to the matching problem.

The interior line element is

$$
d s_{-}^{2}=-d t^{2}+a^{2}(t)\left(d \rho^{2}+\Upsilon_{, \rho}^{2}(\rho, \epsilon) d x^{2}+\Upsilon^{2}(\rho, \epsilon) d \varphi^{2}\right)
$$

and the exterior line element is

$$
d s_{+}^{2}=-e^{2(\gamma-\psi)}\left(d T^{2}-d R^{2}\right)+e^{2 \psi} d Z^{2}+R^{2} e^{-2 \psi} d \Phi^{2} .
$$

By a collapsing RW universe, we mean one for which the scale factor $a(t)$ decays to zero in finite time;

$$
\lim _{t \rightarrow 0^{-}} a(t)=0,
$$

where by a time translation we have set the time of complete collapse to be at $t=0$. Of course this includes the dust model considered above. Note that since we have dropped the junction condition $\left[K_{\mu \nu}\right]=0$, the matching condition (11) no longer holds, and so we are not restricted to dust. Metric continuity across the comoving hypersurface $\rho=\rho_{0}$ yields

$$
\Upsilon_{, \rho}(\rho, \epsilon) a(t) \stackrel{\Sigma}{=} \exp \left(\psi\left(R_{0}(T), T\right)\right)
$$

where

$$
\left.\Upsilon_{, \rho}(\rho, \epsilon)\right|_{\Sigma}= \begin{cases}\cosh \rho_{0}, & \epsilon=-1, \\ 1, & \epsilon=0, \\ \cos \rho_{0}, & \epsilon=+1 .\end{cases}
$$

We note that if $\rho_{0}=\frac{\pi}{2}$ in the case $\epsilon=+1$, then the matching conditions are violated. So we rule out this case. Noting then that $\left.\Upsilon_{, \rho}(\rho, \epsilon)\right|_{\Sigma} \neq 0$, we immediately obtain

$$
\lim _{T \rightarrow T_{*}} \psi\left(R_{0}(T), T\right)=-\infty
$$

where

$$
T_{*}:=\lim _{t \rightarrow 0^{-}} T_{0}(t)
$$

where $T_{0}(t)$ is the solution of the metric matching condition

$$
\left(\frac{d T_{0}}{d t}\right)^{2} \stackrel{\Sigma}{=} e^{-2(\gamma-\psi)}\left(1-\left(R_{0}^{\prime}\right)^{2}\right)^{-1} .
$$

Now $\psi$ satisfies the linear wave equation in 3-dimensional Minkowski space-time (A.6), the solution of which can be written in the integral form

$$
\begin{aligned}
\psi(T, x, y)= & \frac{1}{2 \pi} \frac{\partial}{\partial T}\left\{\int_{S(T)} \frac{\psi_{0}\left(x^{\prime}, y^{\prime}\right)}{\left[T^{2}-\left(x-x^{\prime}\right)^{2}-\left(y-y^{\prime}\right)^{2}\right]^{1 / 2}} d x^{\prime} d y^{\prime}\right\} \\
& +\frac{1}{2 \pi} \int_{S(T)} \frac{\psi_{1}\left(x^{\prime}, y^{\prime}\right)}{\left[T^{2}-\left(x-x^{\prime}\right)^{2}-\left(y-y^{\prime}\right)^{2}\right]^{1 / 2}} d x^{\prime} d y^{\prime}
\end{aligned}
$$


where

$$
S(T)=\left\{(T, x, y): T^{2} \geq\left(x-x^{\prime}\right)^{2}+\left(y-y^{\prime}\right)^{2}\right\}
$$

and

$$
\psi_{0}:=\left.\psi\right|_{T=0}, \quad \psi_{1}=\left.\frac{\partial \psi}{\partial T}\right|_{T=0}
$$

are Cauchy initial data set on an arbitrary initial time slice (which we label as $T=0)$. We assume that these initial data are finite in an appropriate sense. Imposing smoothness and compact support are sufficient, although more general data would also satisfy our requirements [20]. This forms part of our assumption that all initial data for the problem are regular. Then the solution (32) obeys an a priori bound which holds for all finite $T>0$ [2, 20]. Hence for any $T_{1}>0$

$$
\left|\psi\left(R, T_{1}\right)\right|<+\infty, \quad R \geq 0 .
$$

So if $T_{*}<+\infty$, the limit equation (31) cannot be satisfied.

A similar conclusion holds in the case that $T_{0}=+\infty$. We can expand (32) in inverse powers of $T$ to obtain a uniformly convergent series representation [20]

$$
\psi(R, T)=\sum_{k=1}^{\infty} \frac{\psi_{k}(R)}{T^{k}},
$$

which yields $\lim _{T \rightarrow \infty} \psi(R, T)=0$ uniformly in $R$ for all $R \geq 0$. Hence (31) cannot be satisfied in this case, and so metric matching is ruled out.

\section{Null expansions}

We can also apply to the cylindrical case a result of Fayos, Senovilla and Torres [21], that if we have two $C^{3}$, orientable, space-times, $\mathcal{U}^{-}$and $\mathcal{U}^{+}$carrying $C^{2}$ metrics $g^{-}$and $g^{+}$ respectively, then every quantity in the resultant matched space-time $\mathcal{U}^{4}$ constructed from the metric, its first derivatives and some $C^{1}$ tensor fields must be continuous across the boundary. In the spherically symmetric case the null geodesic congruences are invariantly defined so the signs of the expansion scalars of these congruences must be continuous across the boundary.

The ingoing radial null geodesics of the interior space-time $\mathcal{V}_{-}$are given by

$$
l_{-}^{\mu}=\frac{1}{a(t)} \delta_{t}^{\mu}+\frac{1}{a(t)^{2}} \delta_{r}^{\mu}
$$

with expansion scalar

$$
\theta_{-}=\nabla_{\mu} l_{-}^{\mu}=\frac{2 \dot{a}}{a^{2}}+\frac{1}{\rho a^{2}} .
$$

The ingoing radial null geodesics of the exterior space-time $\mathcal{V}^{+}$have expansion scalar

$$
\theta_{-}=\frac{b(U)}{2 R} e^{-2(\psi-\gamma)} \quad \text { for some } \quad b(U)>0 .
$$

We can conclude that, in $\mathcal{V}^{-}$, due to the t-dependance in the scale factor $a(t)=a_{0}|t|^{2 / 3}$ as $-t \rightarrow 0, \theta_{-} \rightarrow-\infty$ whereas $\mathcal{V}^{+}$has $\theta_{-}$strictly positive. The 
discontinuity in the sign of $\theta$ across the boundary is in agreement with [21]. We could equivalently show that the region $\mathcal{V}^{-}$does display the formation of trapped surfaces $\theta=\theta_{+} \theta_{-}>0$ whereas the region $\mathcal{V}^{+}$does not.

\section{Conclusions and Discussion}

We summarize the above as follows:

Proposition 1 Let $\left(\mathcal{V}^{+}, g^{+}\right)$be a vacuum cylindrically symmetric space-time with metric described by ([6), and with the following assumptions:

(i) In $\mathcal{V}^{+}$the metric function $\omega$ is analytic,

(ii) In $\mathcal{V}^{+}$the metric function $\psi$ has regular initial data,

(iii) $\mathcal{V}^{+}$contains no trapped surfaces initially and is not radially closed.

Let $\left(\mathcal{V}^{-}, g^{-}\right)$be a Robertson Walker space-time with the energy conditions $\rho>0$ and $\rho+p \geq 0$. Let $\left(\mathcal{V}^{+}, g^{+}\right)$and $\left(\mathcal{V}^{-}, g^{-}\right)$be matched across a $C^{2}$ hypersurface $\Sigma$ with continuous normal $\vec{n}$ pointing out of $\mathcal{V}^{-}$and into $\mathcal{V}^{+}$. Then at some value of the cosmological time and for all subsequent times the matching breaks down.

This result demonstrates the impossibility of the existence of an isotropic, cylindrically symmetric star, that evolves from a regular initial state (or even a star with a cylindrically symmetric portion). Matching may be possible up until a trapped surface forms in $\mathcal{V}^{-}$at a time $t=t^{*}$. By rearrangement of the matching conditions, we can show that $t^{*}$ is given by the largest value of $t$ for which

$$
\left(R_{0}^{\prime}\right)^{2}<1
$$

where

$$
\left(R_{0}{ }^{\prime}\right)^{2} \stackrel{\Sigma}{=} 4 \dot{a}^{2}\left(\frac{\Upsilon}{\Upsilon_{, \rho}}\right)^{2} .
$$

In the time up until $t^{*}$, matching of the two space-times may be possible. However the initial conditions necessarily imply evolution to a state where matching is not possible.

A spherically symmetric static vacuole in a dust RW cosmology was shown to be possible [22] and it was deduced that the observed cosmological expansion would not affect local physics on astrophysical scales. However it then became clear that a more general model was needed, other geometries for the interior region, and especially for the shape of the boundary, should be considered. Senovilla and Vera [23] proved that embedding a cylindrically symmetric static region in an expanding RW cosmology is always impossible irrespective of the matter inside the cavity. Mars [24, 25] investigated the Einstein-Straus model with a general static cavity embedded in a RW cosmology and obtained the result that the boundary of the static region must be a 2-sphere and that for various reasonable energy momentum tensors the interior is also spherically symmetric. We can consider the complementary matching of a cylindrically symmetric vacuum interior with a RW exterior and impose regularity on the axis of a vacuum 
interior without affecting the matching, i.e. the axis is not singular. Matching of these two space-times may be possible for a finite amount of time up until a trapped cylinder appears in the RW exterior. This leads to a contradiction and prevents the matching from persisting and so again, we do not have a valid physical configuration. Since our results also hold taking the vacuum region to be the interior and the RW the exterior, they complement [23] by also ruling out a dynamical cylindrically symmetric vacuum interior.

In light of these results the impossibility of a cylindrical isotropic star is perhaps unsurprising. However the purpose of this study is to obtain a clearer picture of simple non-spherical, and more specifically, cylindrically symmetric systems in General Relativity. In ongoing work, we are studying cylindrical collapse for other matter models.

\section{Acknowledgement}

The authors wish to thank Filipe Mena for discussions and Raul Vera for his careful reading of this paper and for his very helpful and enlightening comments. This research is supported by Enterprise Ireland grant SC/2001/1999.

\section{Appendix A.}

The $G_{4}^{3}$ field equation component for unpolarized vacuum space-time (6):

$$
\begin{aligned}
\frac{\partial^{2} \omega}{\partial R^{2}}-\frac{\partial^{2} \omega}{\partial T^{2}}= & \frac{3 \omega e^{4 \psi}}{2 \alpha^{2}}\left(\left(\frac{\partial \omega}{\partial R}\right)^{2}-\left(\frac{\partial \omega}{\partial T}\right)^{2}\right)+\frac{1}{\alpha} \frac{\partial \alpha}{\partial R}\left(\frac{\partial \omega}{\partial R}-4 \omega \frac{\partial \psi}{\partial R}\right)+ \\
& +2 \omega\left(\frac{\partial \psi^{2}}{\partial R}-\frac{\partial \psi^{2}}{\partial T}+\frac{\partial^{2} \gamma}{\partial R^{2}}-\frac{\partial^{2} \gamma}{\partial T^{2}}-2 \frac{\partial^{2} \psi}{\partial R^{2}}+2 \frac{\partial^{2} \psi}{\partial T^{2}}\right)+ \\
& +\frac{4 \omega}{\alpha}\left(\frac{\partial \alpha}{\partial T} \frac{\partial \psi}{\partial T}\right)-4\left(\frac{\partial \omega}{\partial R} \frac{\partial \psi}{\partial R}-\frac{\partial \omega}{\partial T} \frac{\partial \psi}{\partial T}\right)-\frac{1}{\alpha} \frac{\partial \alpha}{\partial T} \frac{\partial \omega}{\partial T} \cdot(\mathrm{A} .1)
\end{aligned}
$$

Field equations for polarized vacuum space-time $\alpha \neq R(\underline{19})$ :

$$
\begin{array}{ll}
\frac{\partial^{2} \alpha}{\partial R^{2}}-\frac{\partial^{2} \alpha}{\partial T^{2}} & =0 \\
\frac{\partial \alpha}{\partial R} \frac{\partial \psi}{\partial R}-\frac{\partial \alpha}{\partial T} \frac{\partial \psi}{\partial T}+\alpha\left(\frac{\partial^{2} \psi}{\partial R^{2}}-\frac{\partial^{2} \psi}{\partial T^{2}}\right) & =0 \\
\left(\frac{\partial \psi}{\partial R}\right)^{2}-\left(\frac{\partial \psi}{\partial T}\right)^{2}+\frac{\partial^{2} \gamma}{\partial R^{2}}-\frac{\partial^{2} \gamma}{\partial T^{2}} & =0 \\
\frac{\partial \gamma}{\partial R} \frac{\partial \alpha}{\partial T}+\frac{\partial \alpha}{\partial R} \frac{\partial \gamma}{\partial T}+\frac{\partial^{2} \alpha}{\partial T \partial R}\left(\left(\frac{\partial \alpha}{\partial T}\right)^{2}-\left(\frac{\partial \alpha}{\partial R}\right)^{2}\right) & =0
\end{array}
$$

Field equations for polarized vacuum space-time $\alpha=R(29)$ :

$$
\begin{array}{ll}
\frac{\partial^{2} \psi}{\partial T^{2}}-\frac{1}{R} \frac{\partial \psi}{\partial R}-\frac{\partial^{2} \psi}{\partial R^{2}} & =0 \\
\frac{\partial \gamma}{\partial T}-2 R \frac{\partial \psi}{\partial R} \frac{\partial \psi}{\partial T} & =0
\end{array}
$$


On isotropic cylindrically symmetric stellar models

$$
\begin{aligned}
& \frac{\partial \gamma}{\partial R}-R\left(\left(\frac{\partial \psi}{\partial T}\right)^{2}+\left(\frac{\partial \psi}{\partial R}\right)^{2}\right)=0 \\
& \frac{\partial^{2} \gamma}{\partial R^{2}}-\frac{\partial^{2} \gamma}{\partial T^{2}}-\left(\frac{\partial \psi}{\partial T}\right)^{2}+\left(\frac{\partial \psi}{\partial R}\right)^{2}=0
\end{aligned}
$$

\section{References}

[1] Thorne K S 1972 in Magic without Magic: John Archibald Wheeler, edited by J. Klauder (Freeman, San Francisco) Box 32.3.

[2] Berger B K, Chrusciel P T and Moncrief V 1995 Ann. Phys. (N.Y.) 237 322-354.

[3] Apostolatos T A and Thorne K S 1992 Phys. Rev. D46 2435.

[4] Echeverria F 1993 Phys. Rev. D47 2271.

[5] Singh T P and Vaz C 2003 "Gravitational collapse of an infinite dust cylinder" gr-qc/0304076

[6] Nakao K-I and Morisawa Y 2002 "High speed dynamics of collapsing cylindrical dust fluid" gr-qc/0212089

[7] Goncalves S and Jhingan S 2002 Int. J. Mod. Phys. D11 1469-1478.

[8] Nolan B C 2002 Phys. Rev. D65 104006.

[9] Periera P R and Wang A 2000 Phys. Rev. D62 124001.

[10] Wang A 2003 Phys. Rev. D68 064006.

[11] Goncalves S 2000 Phys. Rev. D62 124001.

[12] Oppenheimer J R and Snyder H 1939 Phys. Rev. 56455.

[13] Mars M and Senovilla J M M 1993 Class. Quantum Grav. 10 1865-1897.

[14] Kramer D, Stephani H, MacCallum M A H, Hoenselaers C and Herlt E 2003 Exact Solutions of Einstein's Field Equations Cambridge University Press.

[15] Carot J, Senovilla J M M and Vera R 1999 Class. Quantum Grav. 16 3025-3034.

[16] Barnes A 2000 Class. Quantum Grav. 172605.

[17] Barrabès C, Israel W and Letelier P S 1991 Phys. Lett. A 16041.

[18] Vera R 2002 Class. Quantum Grav. 19 5249-5264.

[19] Thorne K S 1965 PhD thesis, Princeton University, University Microfilms Inc., Ann Arbor, MI.

[20] Ashtekar A, Bicak J and Schmidt B G 1997 Phys. Rev. D55 669.

[21] Fayos F, Senovilla J M M and Torres R 1996 Phys. Rev. D54 4862.

[22] Einstein A and Straus E G 1945 Rev. Mod. Phys. 17120.

[23] Senovilla J M M and Vera R 1996 Phys. Rev. Lett. 782284.

[24] Mars M 1998 Phys. Rev. D57 3389-3400.

[25] Mars M 2001 Class. Quantum Grav. 18 3645-3663. 